\title{
Embryogenesis Explained. By Natalie K. Gordon and Richard Gordon. World Scientific: Hackensack, NJ, USA, 2016; 784 pp.; SGD 281; ISBN: 978-981-4350-48-8
}

\author{
Palmiro Poltronieri \\ National Research Council of Italy, AgroFood Department, Institute of Sciences of Food Productions, \\ Lecce 73100, Italy; palmiro.poltronieri@ispa.cnr.it
}

Received: 7 July 2017; Accepted: 7 July 2017; Published: 10 July 2017

In Fall, 2016 a new book on embryo development at the molecular and cellular level of comprehension was released by World Scientific Publishing, "Embryogenesis Explained". The authors, Natalie K. Gordon, retired from the University of Manitoba, Canada, and Richard Gordon, from Wayne State University, and Gulf Specimen Aquarium and Marine Laboratory, USA, are eminent scientists that over their long-standing achievements in the field of embryogenesis, have proposed novel insights into the mystery of life, i.e., how a single fertilised egg develops into a fully functioning, multi-cellular organism. This book reviews the publications by the scientific community and compares the hypotheses with those proposed by the two authors: they proposed and then supported their model of the hierarchical genome and the cell state splitter (firstly introduced in 1993), describing and supporting the effects and the role of differentiation waves. The model discussed by the authors is based on a simple, unifying idea that differentiation waves, based on cell cytoskeleton, i.e., contraction and expansion of the network of microfilaments and microtubules, is at the basis of mechano-transduction signalling, that brings transcription factors to regulate chromatin into specific cell-stage differentiation states, based on regulons, i.e., the wholeness of opened DNA regions and inaccessible DNA regions. Thus, from determination of a cell's fate, i.e., signaling to advancement of process, to differentiation state, i.e., new transcriptional program and reprogramming of active regulons, clusters of cells thus divide, mature and form the various cell type component tissues of the developing embryo.

In the sixties, some scientists proposed explanations for embryo development, among others Antone Jacobson, who proposed inductive processes leading to gradual cumulative effects of interactions among embryonic tissues. The authors here review most of the theories proposed in the past twenty years, and discuss how the theories intertwine with their own ideas of the differentiation waves: through their observations at the microscope they succeeded to document extensively, reaching a unified model, the cell state splitter, summarised in their review in 2016 in Theoretical Biology and Medical Modelling. The differentiation waves are well documented, observed through time-lapse movie sequences followed by numbering of each wave, in consecution order, as Contraction 1, Expansion 1, Contraction 2 (CEC) and so on.

As they discuss on page 644: "At each stage of differentiation, a bistable cell state splitter is triggered by mechanical signals to cause an expansion or a contraction wave", that ...coordinates clusters of cells (contracting or expanding their fibrils) so that the signal is transferred through the sheets of cells, that tend to differentiate together.

The extremely detailed information presented in the book will be extraordinarily useful to graduate students and post-docs in the fields of Embryology, Biology, Biochemistry, Molecular Biology, Zoology, Comparative anatomy, and Histology, where the Embryology notions are provided for embryo development in many species to compare peculiarities and universality of mechanisms of tissues development, introducing similarities and differences among the various vertebrates and invertebrates. More precisely, the book explains how scientific research is based on understanding 
mechanisms and searching for data that can explain and support models, theories, and new hypothesis. The simplification of cell function based on DNA and proteins as the main actors making the cell functioning, while RNA was there only to pass the information to proteins, has been recognised as false in the first decade of this century. DNA regulatory regions, non-coding RNAs, and the stratified layers at the basis of regulation of transcription and translation have come out through projects such as ENCODE, with more and more genes codifying for RNAs, in respect to the number of protein coding genes. It is thus my great pleasure to recognise with these authors the high value of information they provided on the ways DNA is organised in euchromatin and heterochromatin regions, and how these can be switched on and off by regulatory signals, arriving from contraction or expansion waves, releasing proteins and transcription factors (the new cell determination state) that modify the DNA topology and also the epigenetic states (the new differentiation state or new regulon).

The authors provide an overview on the several types of second messengers and signaling pathways, including the inositol polyphosphates (IPn, PIP) and their kinases and phosphatases, the role of these ligands as allosteric activators and scaffold for events in the cytoplasm and even in the nuclei. The description of the protein kinases is even more comprehensive and deeply detailed.

The chapters are: Chapter 1: How embryogenesis began in evolution, Chapter 2: Development anatomy of the Axolotl, Chapter 3: Developmental genetics, Chapter 4: Epigenetics. Higher order gene control, Chapter 5: The cytoskeleton, Chapter 6: The cell state splitter and differentiation waves, Chapter 7: The differentiation tree and the fate map of the Axolotl, Chapter 8: Signal transduction from the cell state splitter to nuclear state splitter, Chapter 9: The nuclear state splitter, Chapter 10: Irritable protoplasm: forerunners to differentiation, Chapter 11: Why evolution is progressive, Chapter 12: Wholeness and the implicate embryo: embryogenesis as self-construction of the observer.

This book is a great textbook on the salamander, Axolotl, Ambystoma mexicanum, one of the model animals studied for embryogenesis. The authors describe two type of scientists: those who, for the fast reproduction and quick developmental process prefer to study organisms such as the fruitfly, Drosophila melanogaster, to parasitic wasps, to Xenopus laevis frogs, and those who prefer to document the longer, time-requiring developmental stages in embryogenesis of more complex animals as general, universal processes of embryo development.

The first information that catches the reader is the extremely great size of the Axolotl genome, ten times more than in humans. It means that regulatory DNA regions are more spread and impart more complex regulation, and add features to salamanders, i.e., also in Necturus maculosus, in which the ratio with human DNA is 34:1. One of their particularities is the ability to regenerate and reform tissues that were severed. Regeneration is one of the main frontiers of developmental biology. The book tries to explain the presence of stem cells, using the example of a mitotic cell that forms a more differentiated, bigger daughter cell and a smaller cell conserving the stemness. The authors introduce the two types of embryos present in nature, the mosaic embryo, that has unequal divisions that determine the fate of the daughter cells, and the regulating embryos, that form and develop from a single cell and undergo rounds of equal cell divisions.

The implications in genetic diseases such as the "spina bifida" are presented, with insights on the perfect or less perfect closure of the endodermal cells in the neural crest.

Overall, the book is a great book, documenting with images, schematic reproductions and drawings the embryo development, showing parallelisms, universal processes and the peculiarities of various vertebrates and invertebrates. The book also introduces the embryogenesis processes in plants, although at a more general level to make parallelisms and discuss the universality of the process. The final chapter introduces holistic and wholeness concepts, through the quantum physics formulae, such as the quantum entanglement and the observer effect, using those to describe the relationship among the cell sheets and tissues in the cybernetic embryo.

The book is extraordinarily rich in illustrations, the major part of data acquired by the authors and their students, and those from other sources are all acknowledged and cited in figure captions. Therefore, the title Embryogenesis Explained could well be interpreted as Embryogenesis Illustrated. 
But here the term Explained is more accurate in the fact that all the step-by-step processes either at molecular level either at supramolecular level (cell layers, tissues) are questioned: hypotheses can be proven false or potentially true by observations, collecting data, finding universal mechanisms, i.e., applying the scientific method analysis. There are still unsolved problems and possible solutions, in support of models and hypotheses, that require further testing, for the next generation of researchers in search of simple and universal mechanisms governing the embryo development.

(C) 2017 by the author. Licensee MDPI, Basel, Switzerland. This article is an open access article distributed under the terms and conditions of the Creative Commons Attribution (CC BY) license (http://creativecommons.org/licenses/by/4.0/). 